\title{
A Study of Long-term Changes in Summer Infection Levels of Japanese Flounder Paralichthys olivaceus with the Monogenean Neoheterobothrium hirame in the Central Sea of Japan, with an Application of a New Technique for Collecting Small Parasites from the Gill Filaments
}

\author{
Hilal Anshary ${ }^{1}$, Kazuo Ogawa* ${ }^{1 *}$, Masahito Higuchi ${ }^{2}$ and Tetsuo Fujii ${ }^{3}$ \\ ${ }^{1}$ Department of Aquatic Biosciences, Graduate School of Agricultural and Life Sciences, \\ The University of Tokyo, Yayoi, Bunkyo, Tokyo 113-8657, Japan \\ ${ }^{2}$ Aquaculture and Biotechnology Division, Niigata Prefectural Fisheries and Marine \\ Research Institute, Ikarashi, Niigata 950-2171, Japan \\ ${ }^{3}$ Japan Sea National Fisheries Research Institute, Suido-cho, \\ Niigata 951-8121, Japan
}

(Received December 19, 2000)

\begin{abstract}
Neoheterobothrium hirame infection of 0-year wild Japanese flounder Paralichthys olivaceus was investigated. This study was based on annual samples (preserved in formalin or alcohol) totalling 316 fish caught off Igarashi-hama in July and/or August of 1989-1993 and 505 fish caught off Murakami City in August of 1993-1999, Niigata Prefecture. A stirring method was developed to collect the monogenean from the fixed gill filaments. All the parasites were dislodged from the fixed gill filaments by stirring gills of individual hosts in $150 \mathrm{~mL}$ of water with a magnet $(40$ $\mathrm{mm}$ long and $8 \mathrm{~mm}$ in diameter) at 1150-1200 rpm for $20 \mathrm{~min}$ and $30 \mathrm{~min}$ for the 0-year fish and 1year fish, respectively. Adult parasites were macroscopically observed on the buccal cavity wall, whereas immature ones on the gill arches and rakers were detected under a stereomicroscope. No N. hirame was found from 1989 to 1992 . The earliest records were from samples caught off Igarashi-hama and Murakami City in 1993, though the prevalence of infection was very low. The parasite occurrence changed annually in the Murakami samples. The annual fluctuations may have been influenced by the infection level of co-existing 1-year fish and the population size of 0-year fish recruited each year. It is inconclusive whether or not the monogenean is an indigenous parasite of Japanese flounder in this area.
\end{abstract}

Key words: Neoheterobothrium hirame, Paralichthys olivaceus, Japanese flounder, Monogenea, infection level, ecology

Infection of Neoheterobothrium hirame has been reported from both cultured and wild Japanese flounder Paralichthys olivaceus (see Michine, 1999). The parasite has spread rapidly among the flounder populations in Japanese waters and recently it has been reported from various localities in Japan (Ogawa, 1999). The sharp decline in the catch of wild Japanese flounder has been linked to some possible causes of mortality. For

\footnotetext{
* Corresponding author

E-mail: aogawak@mail.ecc.u-tokyo.ac.jp
}

instance, Furuta (1999) postulated that the scarcity of food leading to a high risk of predation of starved fish might account for this decline. Flounder populations have been diagnosed to suffer from anemia which may also contribute to mortality of wild Japanese flounder. Miwa and Inouye (1999) suspected the cause to be virus-like particles in the hematopoietic tissue of anemic fish, but no definitive conclusion has been reached. Yoshinaga et al. (2000a) attributed anemia to hematophagia by the monogenean $N$. hirame. Since $N$. hirame has become widespread, it may have an impact on fish 
condition sufficient to lead to mortality in wild populations (Ogawa, 1999). Although it is possible to eliminate immature worms on the gill filaments by bathing infected fish in seawater to which extra sodium chloride has been added (Yoshinaga et al., 2000b), no drugs have so far been available to eradicate adult parasites embedded posteriorly in the buccal cavity wall of cultured fish. In spite of a possible impact of the parasite on wild flounder populations, very few studies on the biology of $N$. hirame (see Yoshinaga et al., 2000c) and the occurrence of the parasite on wild flounder populations have been conducted.

Traditionally, gills are directly observed under a stereomicroscope to find and count monogeneans. Neoheterobothrium hirame has been found on the gill filaments, arches and rakers and in the buccal cavity wall of the host (Ogawa, 1999). Parasites were easily detected on the gill arches/rakers and on the buccal cavity wall, but it was not the case for those on the gill filaments. Newly attached $N$. hirame are only about 0.3 $\mathrm{mm}$ long (Ogawa, 2000) and since they occur between the secondary gill lamellae, the possibility of overlooking these small parasites is high. Even after some growth, medium-sized parasites may be hard to see. Furthermore, it is also time-consuming to detect all recently attached parasites under a stereomicroscope, especially when large numbers of fish are to be examined.

The aims of the present study were to develop a method for the detection of small parasites on the gills of the host, and by using this method, to investigate whether there are long-term annual changes in the infection levels of $N$. hirame in wild flounder populations.

\section{Materials and Methods}

\section{Standardization of stirring method}

We developed a new method to accurately and rapidly quantify the number of parasites on the gill filaments, using a magnetic stirrer to dislodge parasites from formalin- or alcohol-fixed material. Detached parasites were then collected from the sediment and counted under a stereomicroscope. This method needed to be standardized before it was applied practically. A multiple-type magnetic stirrer (HS-360, luchi Co., Ltd., Osaka) was used, and stirring speed was measured by a stroboscope (Testo SR-475, Germany). The first step was to determine an appropriate volume of tap water to be used in glass beakers and to identify a suitably sized magnet, so that tests could be run at different speeds of rotation. The second step was to measure the dislodgement rate of the parasite from the gill filaments by testing three different speeds: low (490-570 rpm), middle (870-930 rpm) and high (1150-1200 rpm).

The gills of large and small heavily infected flounder (preserved in formalin) obtained from Iwate Prefecture and Mie Prefecture, respectively, were used to examine the dislodgement rate of parasites from the gill filaments in relation to time and speed of the stirrer. Prior to stirring, the gills of 1-year fish (large fish; $33.5-38.5 \mathrm{~cm}$ in total length) were cut into several pieces of about $1 \mathrm{~cm}$ long for more efficient dislodgement of the parasites, while the whole gills (separated into 4 arches) were used for 0-year fish (small fish; $10.3-14.3 \mathrm{~cm}$ in total length). After several preliminary trials, a glass beaker (volume: $300 \mathrm{~mL}$ ) containing $150 \mathrm{~mL}$ of tap water and a "PTFE stirring bar" magnet, $40 \mathrm{~mm}$ long and $8 \mathrm{~mm}$ in diameter, was found to be suitable for stirring the gills at these speeds. The gills of large fish were stirred at the three different speeds for 10,20, and $30 \mathrm{~min}$. In the case of smaller fish, only the highest speed of rotation was tested for 5, 10, 15 and $20 \mathrm{~min}$. After treatment, the numbers of parasites dislodged and those still attached to the gills were counted, and the rate of dislodgement was calculated. We used 5 large fish for each stirring speed and 10 small fish for the high speed. The intensity of infection ranged from 13 to 255 in the small fish ( $n$ $=10$ ) and from 31 to 1364 in the large fish $(n=15)$.

\section{Fish samples and parasite examination}

Japanese flounder, originally sampled in two localities in Niigata Prefecture for their biological studies and preserved in formalin or alcohol, were examined for the long-term occurrence of $N$. hirame (Fig. 1). The samples were 0-year fish caught with a small beam trawl at 3-30 $\mathrm{m}$ in depth off Murakami City and at 2-8 $\mathrm{m}$ in depth off Igarashi-hama, designated as the Murakami samples and Igarashi-hama samples, respectively. The distance between the two localities was about 50 $\mathrm{km}$, and the two groups are considered to belong to the same fish population. The Igarashi-hama samples consisted of a total of 316 0-year flounder ( $n=45$ to 84 annually; mean annual body length $3.5 \pm 1.4 \mathrm{~cm}$ S.D. to $5.5 \pm 2.5 \mathrm{~cm}$ S.D.) caught in July and/or August from 1989 to 1993 . The Murakami samples consisted of a total of 505 0-year flounder ( $n=48$ to 102 annually; mean annual body length $6.1 \pm 1.5 \mathrm{~cm}$ S.D. to $8.8 \pm 1.9$ cm S.D.) caught in August each year from 1993 to 1999 and 20 1-year flounder (mean body length $18.5 \pm 1.0 \mathrm{~cm}$ S.D. and $21.0 \pm 3.0 \mathrm{~cm}$ S.D.) caught in 1998 and 1999, respectively.

The method for parasite detection was as follows. The fish were first soaked in tap water to remove the fixatives, and the sediment in the storage vessels was searched under a stereomicroscope for possible detached parasites. No such parasite was found in the sediment. Adult parasites, the posterior parts of which were embedded in the buccal cavity wall, were detected macroscopically. The gills were removed and put in a Petri dish to detect parasites on the gill arches and rakers under a stereomicroscope. After removal of any visible parasites from these habitats, the gills were then transferred to a glass beaker containing $150 \mathrm{~mL}$ tap 


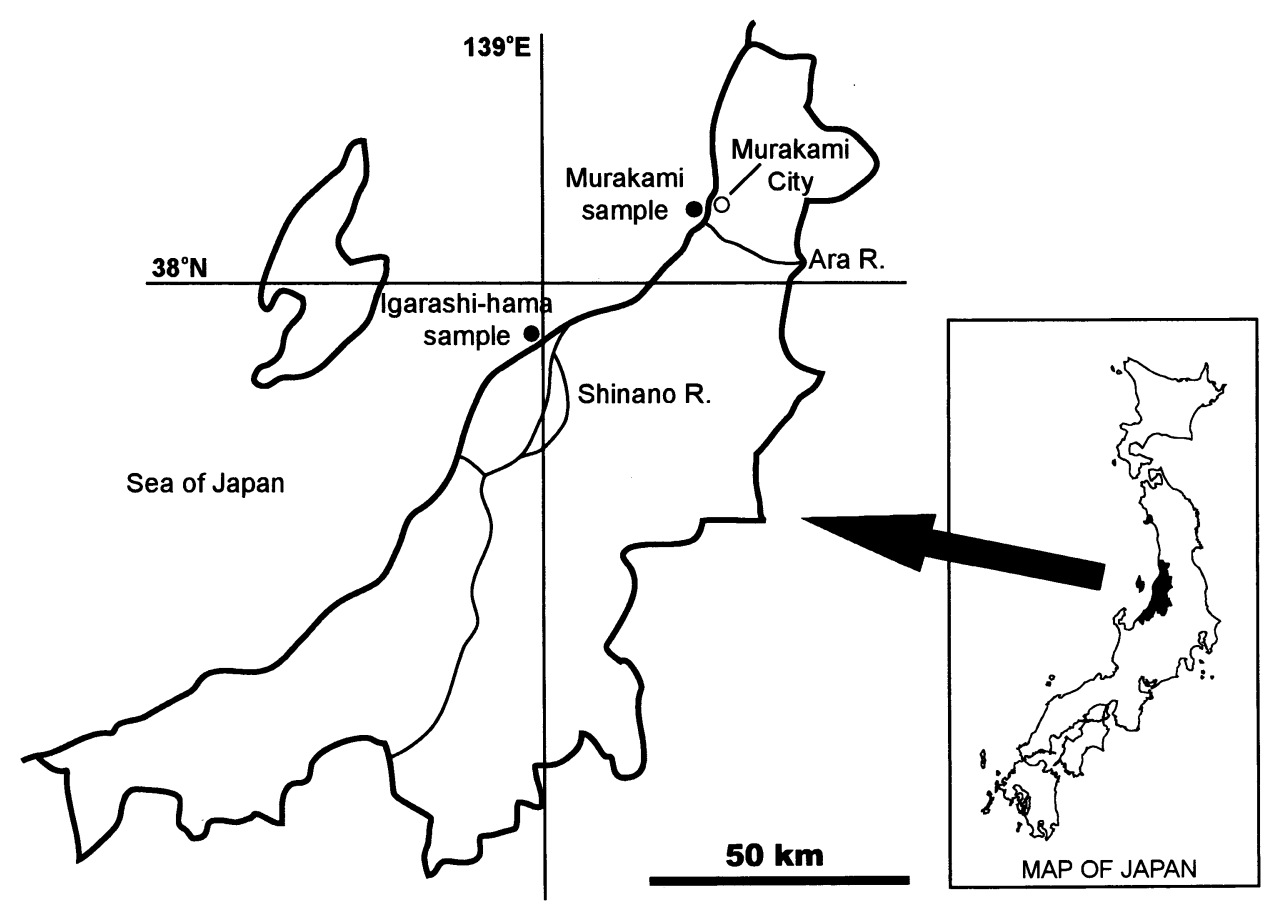

Fig. 1. Map of Niigata Prefecture, showing 2 sampling sites (off Igarashi-hama and off Murakami City).

water, covered with parafilm, and stirred as described above. After decantation of excess water, detached parasites in the sediment were transferred to a Petri dish which was searched under a stereomicroscope. All the parasites found were mounted on slides either in glycerine jelly or in glycerine, and their developmental stages were determined. The developmental stages were based on the number of clamps and the presence or absence of the copulatory organ as follows: Stage $O$ (no clamps), Stage I (1 pair of clamps), Stage II (2 pairs of clamps), Stage III (3 pairs of clamps), Stage IVa (4 pairs of clamps/copulatory organ not visible), Stage IVb (copulatory organ visible).

The ecological terms used follow Bush et al. (1997). Specifically, when we described the infection level of 0 -year and 1-year flounder in Niigata Prefecture, we used "intensity" (the number of parasites per infected fish) for 0-year fish and "abundance" (the number of parasites per host fish regardless of whether or not they are infected) for 1-year fish. "Intensity" was selected for 0 -year fish for the practical reason that the infection level tended to be low. On the other hand, "abundance" was used for 1-year fish, because the infection level of the host fish including uninfected ones is important when we discuss transmission of the parasite from 1-year to 0 year fish.

\section{Data analyses}

Data were analysed by Mann-Whitney U-test or Kruskal-Wallis test (non-parametric tests) using a statistical package, SPSS 9.0 for Windows. $G$ and $G_{\text {adj }}$ tests were performed following Fowler and Cohen (1992).

\section{Results}

\section{Efficacy of stirring on parasite dislodgement}

The efficacy of the stirring method for parasite dislodgement is shown in Fig. 2A for 1-year fish, and in Fig. 2B for 0-year fish. For 1-year fish, an average of $83 \%$ (range $75-91 \%$ ) and $83 \%(72-98 \%$ ) of parasites became detached after stirring for $10 \mathrm{~min}$ at the low and middle speeds, respectively. The detachment rate became higher with additional time, but some did not reach $100 \%$, even after stirring for $30 \mathrm{~min}(93-98 \%$ for the low speed and $94-100 \%$ for the middle speed). At the high speed, on the other hand, some still remained on the gills after 20 minutes with an average of $98 \%$ (96-100\%), but all detached by 30 minutes.

Since the high speed produced the best rate of dislodgement, we tested only the high speed of stirring for the small fish. The range and mean number of parasites dislodged is shown in Fig. 2B. Five min after stirring, the percentage of parasites dislodged ranged from $85 \%$ to $100 \%$ and all parasites had detached after 20 $\mathrm{min}$. Therefore, for large and small fish we chose to stir separated gills in a $300 \mathrm{~mL}$ beaker containing $150 \mathrm{~mL}$ water at high speed (1150-1200 rpm) for 30 and $20 \mathrm{~min}$, respectively.

Annual occurrence of N. hirame on wild 0-year flounder

In the Igarashi-hama samples, no parasites were found on flounder caught from 1989 to $1992(n=250)$. The parasite was first recorded from 3 out of 66 fish examined in 1993 with a mean intensity of one. The parasites were from the secondary gill lamellae and they 

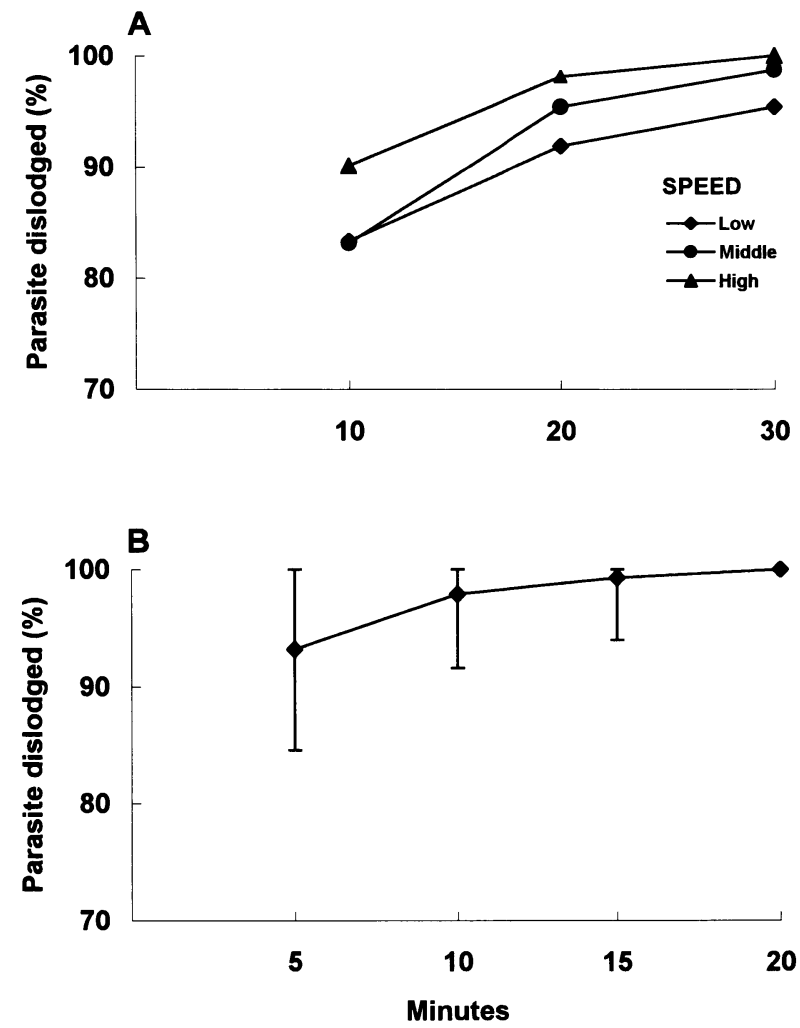

Fig. 2. Dislodgement of Neoheterobothrium hirame from the gill filaments of Japanese flounder using a magnetic stirrer. A: 1-year fish (Total length $=33.5-38.5 \mathrm{~cm}, \mathrm{n}$ $=5$ for each speed), stirrer speed: Low (490-570 rpm), Middle (870-930 rpm), and High (1150-1200 rpm). B: 0-year fish (Total length $=10.3-14.3 \mathrm{~cm}, \mathrm{n}$ $=10$ ), stirrer speed: $1150-1200 \mathrm{rpm}$. Bar indicates range of parasites dislodged in the small fish.

all were in an early stage (stage I). In the Murakami samples, the prevalence and mean intensity of infection of $N$. hirame was not uniform annually $(\mathrm{G}=216.175, P<$
0.001 for the prevalence; Kruskal-Wallis test, $\mathrm{K}=$ 54.596, $P<0.001$ for the mean intensity). In 1993 the prevalence was very low, but it rose significantly in 1994 (Fig. 3). From 1994 to 1996, the prevalence remained high. In 1997 and 1998, the prevalence and mean intensity of infection reached their peak and decreased again in 1999 (Fig. 3). The pattern of infection in different microhabitats showed that more parasites were found on the gill filaments than in the other habitats, except when the prevalence of infection was low in the years 1993 and 1999 (Fig. 4A). A high proportion of parasites were in early developmental stages (stage 0 and I) from 1994 to 1998 , while no early stages were found in either 1993 or 1999 (Fig. 4B).

\section{Prevalence and mean abundance of infection of 1-year flounder}

The prevalence and mean abundance of adult parasites were significantly higher in 1998 than in $1999\left(G_{a d j}\right.$ $=6.754, P<0.05$; Mann-Whitney $U$-test, $U=2.96, P<$ 0.01 ) (Fig. 5). The number of adult parasites per individual fish ranged from 1 to 21 in 1998 and from 1 to 3 in 1999.

\section{Discussion}

A stirring method has been developed to dislodge small monogeneans rapidly from formalin- or alcoholfixed gills. The method was used in the present study to investigate the presence of Neoheterobothrium hirame on the gill filaments and was particularly useful when a large number of fish was to be examined. The small size of the oncomiracidium of $N$. hirame (see Ogawa, 2000) and the location of recently attached parasites amongst the secondary gill lamellae make them difficult to detect, particularly for gills with much

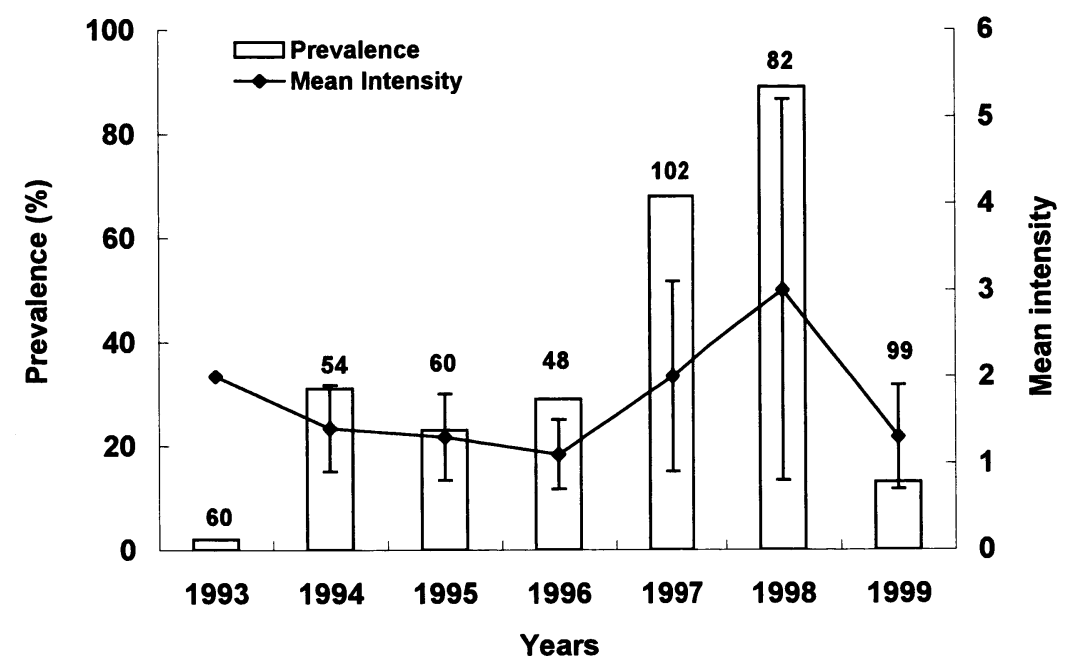

Fig. 3. Annual prevalence and mean intensity of N. hirame infecting 0-year flounder, caught off Murakami City from 1993 to 1999. Bar indicates standard deviation from the mean. The number on the top of each column indicates the number of fish examined. 



Fig. 4. A: Proportion of N. hirame in different microhabitats of Japanese flounder caught off Murakami City. "Unknown" means parasites detached from their habitats when the fish was dissected, bcw = buccal cavity wall, ga/gr = gill arch/gill raker, gf = gill filament. The number on the top of each column indicates the number of parasites.

B: Proportion of developmental stages of $N$. hirame.



Fig. 5. Prevalence and mean abundance of adult N. hirame infecting 1-year flounder caught off Murakami City in 1998 and 1999.

mucus, and thus the possibility of overlooking parasites is high. Using the stirring method with a high speed of stirring for certain periods of time, all parasites on the gill filaments became detached and it was relatively easy to detect these detached parasites in the sediment.

Neoheterobothrium hirame was not present on flounder caught off Igarashi-hama between 1989 and 1992, and it appeared for the first time only in 1993 with a very low prevalence and intensity. This may indicate that the parasite is not indigenous in this area. However, the origin of the parasite is still unknown. Recently, $N$. hirame was reported to be widespread on wild Japanese flounder populations in Japanese waters, except in Hokkaido (Ogawa, 1999).

The occurrence of $N$. hirame on Japanese flounder in Niigata Prefecture changed annually with the infection level increasing from 1993 to 1998 and decreasing again in 1999. The cause of the fluctuations in the prevalence and intensity in 0-year fish was not clear. However, there are some possible factors that might be responsible, such as water temperature, abundance of 0 -year fish and abundance and infection level of older infected fish. Seasonal changes of water temperature are likely to influence parasite development, as for example in Heterobothrium okamotoi (see Ogawa and Inouye, 1997). However, in the present study fish were caught at almost the same time each year when water 
temperatures will be similar. Older fish, mostly 1 -year fish, cohabit with 0 -year fish in the relatively shallow nursery ground in summer (M. Higuchi, pers. com.), and may act as the source of infection of 0-year fish. The more important factors, therefore, in determining summer infection levels of 0 -year fish are the infection level among the 1-year fish and the abundance of 0 -year and 1 -year fish in the nursery ground. It was not easy to estimate the fish population size at a given time.

In summer 1998, the prevalence and abundance of infection of 1-year flounder was high, corresponding with the high infection level of 0 -year flounder. Transmission of parasites to young flounder from these heavily infected older fish may have occurred. In 1999, on the other hand, the prevalence of infection of 0-year fish declined. The proportion of parasites on the gill filaments was lower than in any of the years 1993-1998 and lower than the proportion of parasites in the other habitats in 1999. These observations indicate a low level of new infection in 1999. Even though the prevalence of infection of 1-year fish was relatively high in 1999, the abundance of adult parasites was low at the time of sampling. Besides, the number of flounder fry recorded in this area was at an all-time high; it was estimated that about 10 times as many fry were recruited in 1999 compared with 1998 (M. Higuchi and T. Fujii, pers. com.). Consequently, low levels of parasites available for transmission and the exceptionally large population size of 0 -year flounder may explain the low prevalence of N. hirame on 0-year fish in 1999.

\section{Acknowledgements}

We thank Dr. T. Yoshinaga, National Research Institute of Aquaculture, Mie Prefecture and the Japan Sea-Farming Association, Iwate Prefecture, for kindly providing infected Japanese flounder for the stirring method standardization. We are also thankful to Dr. Craig J. Hayward and anonymous reviewers, for critical reading of the paper.

\section{References}

Bush, A. O., K. D. Lafferty, J. M. Lotz and A. W. Shostack (1997): Parasitology meets ecology on its own terms: Margolis et al. revisited. J. Parasitol., 83, 575-583.

Fowler, J. and L. Cohen (1992): Practical statistics for field biology. John Wiley \& Sons Ltd., Chichester, UK, 227 p.

Furuta, S. (1999): Seasonal changes in abundance, length distribution, feeding condition and predation vulnerability of juvenile Japanese flounder, Paralichthys olivaceus, and prey mysid density in the Tottori coastal area. Nippon Suisan Gakkaishi., 65, 167-174. (In Japanese with an English abstract.)

Michine, A. (1999): Neoheterobothrium sp. found on Japanese flounder cultured commercially or maintained as spawners. Res. Shimane Pref. Center Cult. Fish., 2, 15-23. (In Japanese.)

Miwa, S. and K. Inouye (1999): Histopathological studies of the flounder with anemia found in various places in Japanese coastal waters. Fish Pathol., 34, 113-119. (In Japanese with English abstract.)

Ogawa, K. (1999): Neoheterobothrium hirame sp. nov. (Monogenea: Diclidophoridae) from the buccal cavity wall of Japanese flounder Paralichthys olivaceus. Fish Pathol., 34, 195-201.

Ogawa, K. (2000): The oncomiracidium of Neoheterobothrium hirame, a monogenean parasite of Japanese flounder Paralichthys olivaceus. Fish Pathol., 35, 229-230.

Ogawa, K. and K. Inouye (1997): Heterobothrium infection of cultured tiger puffer, Takifugu rubripes - a field observation. Fish Pathol., 32, 15-20.

Yoshinaga, T., T. Kamaishi, I. Segawa, A. Kumagai, C. Nakayasu, K. Yamano, T. Takeuchi and M. Sorimachi (2000a): Hematology, histopathology and the monogenean Neoheterobothrium hirame infection in anemic flounder. Fish Pathol., 35, 131-136. (In Japanese with an English abstract.)

Yoshinaga, T., T. Kamaishi, I. Segawa and E. Yamamoto (2000b): Effects of NaCl-supplemented seawater on the monogenean, Neoheterobothrium hirame, infecting the Japanese flounder. Fish Pathol., 35, 97-98.

Yoshinaga, T., I. Segawa, T. Kamaishi and M. Sorimachi (2000c): Effect of temperature, salinity and chlorine treatment on egg hatching of the monogenean Neoheterobothrium hirame infecting Japanese flounder. Fish Pathol., 35, 85-88. 